Article

\title{
Information and the Regulation of a Lower Hierarchical Level by a Higher One
}

\author{
Stanley N. Salthe \\ Biological Sciences, Binghamton University, Vestal Parkway East, PO Box 6000, Binghamton, \\ NY 13902-6000, USA; E-Mail: ssalthe@binghamton.edu
}

Received: 2 July 2012; in revised form: 8 October 2012 / Accepted: 9 October 2012 /

Published: 22 October 2012

\begin{abstract}
In this paper I consider the usefulness of the compositional hierarchy model in understanding the information flows involved in group behaviors in animals. I propose that short-term memory can function to transduce information across scale, thereby connecting different modes of information and mediating coherent group motions. This transduction I propose to be mediated by the "sign" as understood in Peircean semiotics, generating the meaning of the information for the social animal.
\end{abstract}

Keywords: compositional hierarchy; semiotics; short term memory; sign

\section{Introduction}

In this paper I will be using the term 'information' to refer to the actual configurations of informational constraints characterizing, or being imposed upon, a system. Such constraints limit, but also enable, system activities and responses. As a simplest, but very clear, physical example, the constants in an equation function as informational constraints upon the dynamics modeled by the equation, while their values are the relevant information. Informational constraints are generally the results of historical contingencies. Information impinging a system from outside would acquire meaning for a system only when it is interpreted by that system as a response, which can be modeled in semiotics discourse, as outlined below in this paper.

Hierarchical forms are today utilized by researchers in different fields, but with little evident understanding of the properties of these structures. In this paper I show how the logical properties of one of these forms - the compositional hierarchy — can be useful in understanding the systems they are applied to. 
In both known forms of hierarchy [1,2] an upper level regulates, controls, interprets or harnesses a lower level under its own regime. In some cases the control is tight, as with "order parameters" [3], in others regulation is loosely imposed by way of boundary conditions [4] as in this study. In a subsumptive hierarchy—e.g., \{physical forces \{chemical attractions $\{$ biological forms\}\} \} - control by the higher level (here, biological) descends through all the levels simultaneously, while in the compositional hierarchy (e.g., [population [organism [cell]]] ) the downward regulation descends only to the next lower level (here, e.g., population to organism), and so is not transitive across the hierarchy [4], but must be transduced at each crossing between levels, converting one mode of information to another [5]. So for example, information in the form received from a population of multicellular organisms will not be felt directly by the cells of an organism in the population, but must be transformed by the organism into another mode of information appropriate for communicating with its cells.

\section{Results and Discussion}

Recently, a paper by Mann et al. [6] made a suggestion concerning cross-scale communication in prawns, the abstract of which I present here:

Inference of interaction rules of animals moving in groups usually relies on an analysis of large scale system behaviour. Models are tuned through repeated simulation until they match the observed behaviour. More recent work has used the fine scale motions of animals to validate and fit the rules of interaction of animals in groups. Here, we use a Bayesian methodology to compare a variety of models to the collective motion of glass prawns (Paratya australiensis). We show that these exhibit a stereotypical 'phase transition', whereby an increase in density leads to the onset of collective motion in one direction. We fit models to this data, which range from: a mean-field model where all prawns interact globally; to a spatial Markovian model where prawns are self-propelled particles influenced only by the current positions and directions of their neighbours; up to non-Markovian models where prawns have 'memory' of previous interactions, integrating their experiences over time when deciding to change behaviour. We show that the mean-field model fits the large scale behaviour of the system, but does not capture fine scale rules of interaction, which are primarily mediated by physical contact. Conversely, the Markovian self-propelled particle model captures the fine scale rules of interaction but fails to reproduce global dynamics. The most sophisticated model, the non-Markovian model, provides a good match to the data at both the fine scale and in terms of reproducing global dynamics. We conclude that prawns' movements are influenced by not just the current direction of nearby conspecifics, but also those encountered in the recent past. Given the simplicity of prawns as a study system our research suggests that self-propelled particle models of collective motion should, if they are to be realistic at multiple biological scales, include memory of previous interactions and other non-Markovian effects.

This provides a good example to examine utilizing a hierarchy format. It is the compositional hierarchy format that is relevant here. Visible in this example as stated are only two levels, but in order to see an example whole in compositional hierarchy format we need at least one other level [4]. In this particular example, that level is implied, and modeled by, memory - that is to say, circuits of neurons in the central nervous system, at lower hierarchical levels than the prawn as a whole [7]. Levels in a 
compositional hierarchy must be separated by dynamical rates of at least an order of magnitude [4], with the highest level undergoing the slowest changes, in this way regulating the effects of the faster changing lower levels by way of imposing relatively unchanging boundary condition constraints upon the lower level system. Information from one level in a compositional hierarchy does not transit directly, or unmediated, to another level. The hierarchy we arrive at in this example is:

\section{[shoal [prawn [neuron]]]}

The shoal is represented locally to a prawn by its immediate neighbors. Through these, the shoal regulates the prawn [8], which regulates its own neuronal activity, which then accumulates upscale via the efferent nervous system to be expressed in the prawn's behavior. In this particular example, memory is found to influence a prawn's behavior relative to other prawns in the shoal. This memory would therefore plausibly be acting as if it were at a larger scale than the prawn itself insofar as it would extend beyond any actual moment in the prawn's existence as a physical object. This functioning short term memory can be represented as the recent and ongoing trajectory of the prawn, which would seem to be an entity at a higher level than an individual prawn at a single moment. This gives us:

\section{[nearest neighbors [prawn’s trajectory [prawn [neurons]]]}

Neurons need to continue to be represented here because it is their activity that generates the experience of the prawn's trajectory. (While the minimal number of levels is three, a compositional hierarchy can have any number of levels, as required by a given application.) The shoal as such has not been replaced, but its effect on the prawn via its nearest neighbors will have been displaced upward by the interpolation of a new level [4,9] between it and the prawn. This synthesized trajectory is itself still being regulated by the shoal by way of the perceived momentary locations of the prawn's neighbors.

This 'prawn's trajectory' construct might be conceived to be an effectively higher level 'meta-stable product' [10] of the neural activities taking place at the lowest level here. It would form a temporary, dynamically maintained, higher level framework whereby the prawn engages its fellows during schooling. Using narrative forms, it could be represented as the present moment actively mediating between the present progressive and present perfect tenses [11]. It could as well be represented in this biological case as the construct:

\section{short-term memory--now--anticipation (SMNA)}

This meta-stable entity is what it seems to me would be represented in Mann et al's model as 'memory', and would be the dynamic actuality of what I am here labeling the 'prawn's trajectory'. It would be synthesized at a lower-than-individual prawn level, but would be enacted and experienced in a way that places it temporally between the prawn and its cohorts, who present to it local input from the shoal. Anticipation here functions to generate agonism in the prawn to the degree that its coherence with the shoal is threatened by increased divergence from the ongoing representation. Since the SMNA can be viewed as being co-constructed by both the shoal as a palpable presence and the prawn, it would effectively be acting semiotically as a sign of the shoal for the prawn [12].

Information in a compositional hierarchy is not transmitted directly from level to level, but must be transduced at each step across scale [13]. We may inquire whether the synthesized trajectory, SMNA, 
is really different in scale from the momentary prawn and from its nearest neighbors. Would it perhaps be better viewed in this hierarchical model as the dynamical process of transduction? I propose that it indeed represents the process of cross-scale transduction itself-as well as signaling the activity of semiosis. To be effective in this example it would need to mediate between two levels of different scale. It more closely matches the scale of the shoal, not in material size, but rather, temporally. It has several times been proposed [4] that difference in rate of activity is the most fundamental basis of scale difference, rather than physical size. The larger are slower, and the smaller an entity is, the faster on average it accelerates. SMNA is effectively larger than the momentary prawn, not in size, but in temporal extension. With regard to the shoal as represented by the prawn's nearest neighbors, SMNA matches them too closely in scale, which is why I think it cannot be taken to be an actual level intermediate between prawn and shoal in a compositional hierarchy. A related criterion of scale is difference in the strength of interactions between levels. The forces within the prawn are much-order of magnitude-stronger, binding it together, than those within the shoal. The neurally generated meta-stable construct, 'prawn's trajectory', clearly cannot fit as an actual level in the hierarchy on this criterion. Thus, if we wish to represent Mann et al's 'memory' here, it would be better to have:

$$
\text { [prawn’s neighbors---SM-[-NA---> prawn [neurons]]] }
$$

with ---[---> representing the process of transduction, whereby Mann et al's 'memory'--my SMNA-- mediates between the shoal and the prawn, constructing feedback information for the prawn about the trajectory of the shoal. I note that Longo and Montévil [14] have proposed that an "extended form of present seems more adequate for the understanding of memory”.

Semiotically, based on the diagrams in Herrmann-Pillath and Salthe [12] and Salthe [15] the understanding that emerges is shown in Figure 1. The inner cycle takes place at the lowest level, within the prawn. 'Interpretance' labels the process of construction and deployment of interpretants, in this case within the nervous system of the prawn. Perception would emerge here from a chain of interpretants deployed at that level-from a process of interpretance. The meaning of the information then emerges at the highest level of the system as coherent swimming with the shoal.

My point in this paper is that, since the prawn and its neighbors (representing the shoal) exist at different scales, a sign is required in order for the shoal to communicate with the prawn. If the sign as it functions here were to be removed, the prawn would react moment by moment only to information from the immediate sense data provided by the shifting positions of its neighbors, with the further complication of needing to choose which neighbor is most important at every moment. Its resulting movements - and, of course, those of its neighbors as well-would become largely incoherent and increasingly uncoordinated, approaching a statistical mechanical process. The sign, because of its perceived larger-than-momentary scale, allows the prawn to track the group as a whole, privileging only anticipated motions that track with prior motions as retained in short-term memory, which would influence anticipation as well, as discussed in detail in Matsuno and Salthe [11]. As support for the view presented in this paper, I note a paper by Zhang and Siller on the physics of short term memory [16]. 
Figure 1. Peircean triadic diagram of semiosis. Neighbors are the object, short-term memory--now--anticipation (SMNA) the sign, the prawn is the system of interpretance [15]. Interpretance here labels the innermost cycle, within the prawn. Arrows indicate direction of information flow. Meaning emerges as coherent swimming.

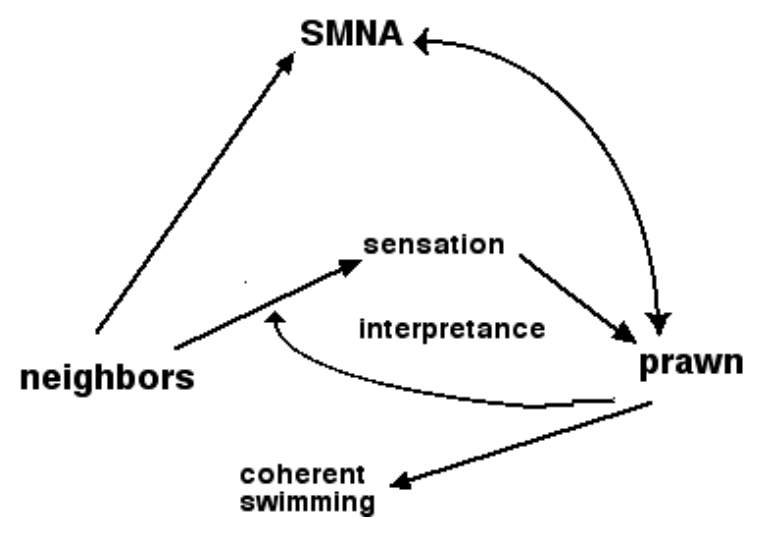

\section{Conclusions}

I provisionally conclude this hierarchy-based semiotic analysis by proposing that generating signs is a function involving short-term memory in animals.

\section{Acknowledgements}

I thank Victoria Alexander and Koichiro Matsuno for perceptive comments.

\section{References}

1. Salthe, S.N. Summary of the principles of hierarchy theory. Gen. Syst. Bull. 2002, 31, 13-17.

2. Salthe, S.N. Hierarchical structures. Axiomathes 2012, 23, 355-383.

3. Haken, H. Information and Self-Organization: A Macroscopic Approach to Complex Systems; Springer: Berlin, Germany, 1988.

4. Salthe, S.N. Evolving Hierarchical Systems: Their Structure and Representation; Columbia University Press: New York, NY, USA, 1985.

5. Lemke, J.L. Opening up closure: semiotics across scales. Ann. New York Acad. Sci. 2000, 901, 100-111.

6. Mann, R.P.; Prna, A.; Strömbom, D.; Garnett, R.; Herbert-Read, J.E.; Sumpter, D.T.; Ward, A.J.W. Multi-scale inference of interaction rules in animal groups using Bayesian model selection. PLoS Comput. Biol. 2012, 8, e1002308, doi:10.1371/journal.pcbi.1002308.

7. Guidolin, D.; Albertin, G.; Guescini, M.; Fuxe, K.; Agnati, L.F. Central nervous system and computation. Quart. Rev. Biol. 2011, 86, 265-285.

8. Bialek, W.; Cavagna, A.; Giardina, I.; Mora, T.; Silvestri, E.; Viali, M.; Walczak, A.M. Statistical mechanics for natural flocks of birds. Proc. Nat. Acad. Sci. 2012, 109, 4786-4791.

9. Salthe, S.N. The spontaneous origin of new levels in a scalar hierarchy. Entropy 2004, 6, 327-343.

10. Matsuno, K.; Salthe, S.N. Chemical affinity as material agency for naturalizing contextual meaning. Information 2012, 3, 21-35. 
11. Matsuno, K.; Salthe, S.N. The origin and development of time. Int. J. Gen. Syst. 2002, 31, 377-393.

12. Herrmann-Pillath, C.; Salthe, S.N. Triadic conceptual structure of the maximum entropy approach to evolution. BioSystems 2011, 103, 315-330.

13. Allen, T.F.H.; Starr, T.B. Hierarchy: Perspectives for Ecological Complexity; University of Chicago Press: Chicago, IL, USA, 1982.

14. Longo, G.; Montévil, M. The inert vs. the living state of matter: extended criticality, time geometry, anti-entropy-An overview. Front. Physiol. 2012, 3, doi: 10.3389/fphys.2012.00039.

15. Salthe, S.N. The System of interpretance: Naturalizing meaning as finality. Biosemiotics 2009, 1, 285-294.

16. Zhang, H.-Y.; Sillar, K.T. The short term memory of motor network performance via activity-dependent potentiation of $\mathrm{Na}+/ \mathrm{K}+$ pump function. Curr. Biol. 2012, 22, 526-431.

(C) 2012 by the authors; licensee MDPI, Basel, Switzerland. This article is an open access article distributed under the terms and conditions of the Creative Commons Attribution license (http://creativecommons.org/licenses/by/3.0/). 